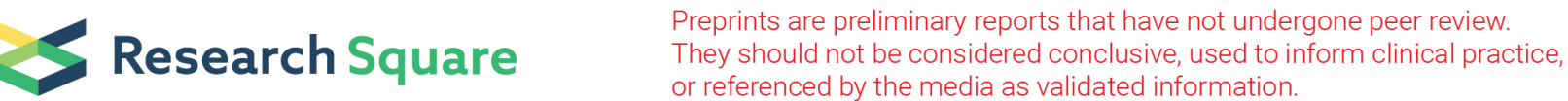

\section{Analysis of the heterogeneity of the BCR H-CDR3 repertoire in the bone marrow and spleen of 3-, 12-, and 20 -month old mice}

Lina Ma

Zunyi Medical University

Xinsheng Yao ( $D$ immunology01@126.com )

Zunyi Medical University

Tao Xinxin

Zunyi Medical University

He Xiaoyan

Zunyi Medical University

Wang Peng

Zunyi Medical University

Ma Long

Zunyi Medical University

Shi Bin

Zunyi Medical University

Research

Keywords: Analysis, heterogeneity of the BCR H-CDR3, repertoire, bone marrow and spleen, mice

Posted Date: November 18th, 2020

DOl: https://doi.org/10.21203/rs.3.rs-27464/v2

License: (9) This work is licensed under a Creative Commons Attribution 4.0 International License.

Read Full License

Version of Record: A version of this preprint was published at Immunity \& Ageing on April 12th, 2021. See the published version at https://doi.org/10.1186/s12979-021-00231-2. 


\section{Abstract}

The number of central and peripheral B cells and their responsiveness are decreased in aged mice. The diversity of mouse central and peripheral $B$ cell repertoires with increasing age has not been elucidated. In this study, we demonstrated that there were significant differences in the usage of some $V, D$, and $J$ genes in the BCR H-CDR3 repertoire of bone marrow B cells, spleen B cells and spleen memory B cells in 3-, 12-, and 20-month-old mice. In the productive, pseudogene, and out-of-frame sequences, bone marrow B cells had significant differences in $5 \mathrm{~J}$ trimming with age; peripheral spleen $\mathrm{B}$ cells and memory $\mathrm{B}$ cells had significant differences in N1 insertion, N2 insertion, P5'D insertion, and 5'D trimming with age. The BCR HCDR3 repertoire diversity of mouse bone marrow $B$ cells, spleen $B$ cells and spleen memory $B$ cells decreased with increasing age. The proportion of overlap in bone marrow and spleen B cells, but not spleen memory B cells, of mice at different ages was lower at 3 months than at 12 and 20 months. This study is the first to report the homogeneity and heterogeneity of the CDR3 repertoire of central and peripheral $B$ cells change as mice age, to further investigation of the decline and response of $B$ cell immunity in young/middle/old-aged mice.

\section{Introduction}

The percentage of B cells and antibody category conversion and reorganization decrease with age[1, 2]. The response $B$ cells produced by aging animals under the same intensity of antigen stimulation are $1 / 10-1 / 50$ that produced by adult animals[3]. B cell clone proliferation changes with age, and the genetic lineage of B cells also undergoes corresponding dynamic changes. In particular, the increase in memory B cell clones is closely related to the immune system status of elderly individuals[4-6].

The diversity of the naive BCR H-CDR3 repertoire is derived from the rearrangement of the germline $V(D) J$ gene in the bone marrow[7] (high-frequency mutations in the periphery that increase the diversity of the $B$ cell repertoire[8] ). A shift of the reading frame during the rearrangement process can result in an out-of-frame sequence, and rearrangement of the $V(D) J$ pseudogene will not produce a functional sequence[9], although an out-of-frame and pseudogene rearrangement failure on one chromosome may be the start $V(D) J$ on another chromosome to continue rearrangement. With the application of HTS to the CDR3 repertoire of T/B cells, the dynamic changes in the body's immune system can be explored by comparing the effective rearrangement of functional genes (in frame) and the rearrangement of the out-of-frame and pseudogene sequences.

Human studies have shown that the lifespan of B cells in elderly individuals is increased, and the production in the bone marrow is reduced[10,11]. B cell expansion clones increase with age[1213]. The repertoire is closely related to changes in age before and after immunization in mice[14]. At present, how the homogeneity and heterogeneity of the CDR3 repertoire of central and peripheral B cells change as mice age is not fully elucidated. 
In elderly individuals, immunoglobulins $\lg M$ and $\lg D$ are reduced, and naive $B$ cells are transformed into memory B cells[12]. Plasma cells produce reduced IgG antibodies, which limit memory B cell diversity[15$16]$. As the mouse age increases, how the corresponding changes in the peripheral memory $B$ cell repertoire has not been elucidated.

In this study, we selected 3-, 12-, and 20-month-old mice and used HTS to detect the bone marrow B cell, spleen B cell, and spleen memory B cell BCR H-CDR3 repertoire. The homogeneity and heterogeneity of the productive, pseudogene, and out-of-frame sequences in the BCR H-CDR3 repertoire were compared and analyzed. The main components and characteristics of the central and peripheral BCR H-CDR3 repertoire of mice at different ages were further studied.

\section{Results}

\section{Preparation of mouse spleen samples and tissue HE staining}

The spleen tissue of mice aged 3, 12, and 20 months was taken (Fig. 1A): the spleen length was approximately $1.5-2 \mathrm{~cm}$, and the color was bright red. The spleen white pulp structure became irregular with increasing age (Fig. 1B). The purity of spleen tissue memory $B$ cells $\left(C D 45 R^{+} C D 27^{+}\right)$by Miltenyi bead sorting was more than $85 \%$ (Fig. 1 C).

\section{Mouse bone marrow B cell, spleen B cell and memory B cell CDR3 repertoire}

The concentration and purity of multiplex PCR products were in accordance with HTS requirements (Fig. S1). The CDR3 repertoire from the HTS of each tissue sample of 3-, 12-, and 20-month-old mice was compared and statistically analyzed according to the productive, pseudogene, and out-of-frame unique and total sequences (Tab. 1).

\section{Gene frequency in the BCR H-CDR3 repertoire}

V gene usage (Fig. 2-1, Fig. S2-1 and Fig. S2-2): (1) Productive sequences: 3-, 12-, and 20-month-old mice were given high-frequency IGHV1-4, IGHV14-1, IGHV1-50, and IGHV1-64. With the change in age, Bone marrow B cell IGHV1-54 and IGHV1-84; spleen B cell IGHV4-1, IGHV1-87, IGHV5-12, and IGHV9-1; and spleen memory B cell IGHV1-4, IGHV5-12-1, and IGHV5-9 showed significant differences at different months $(P<0.05)$. (2) Pseudogene sequences: 3-, 12-, and 20-month-old mice were given highfrequency IGHV1-67 and IGHV1-83. Bone marrow B cell IGHV1-25 and IGHV1-83 and spleen B cell IGHV132 and IGHV1-67 showed significant differences with changes in age $(\mathrm{P}<0.05)$. (3) Out-of-frame sequences: 3-, 12-, and 20-month-old mice were given high-frequency IGHV1-4, IGHV14-1, IGHV150, and IGHV1-64. Bone marrow B cell IGHV11-1 and IGHV14-1; spleen B cell IGHV11-1, IGHV1-4, IGHV187, IGHV1S-45, and IGHV9-1; and spleen memory B cell IGHV1-14, IGHV1-4, IGHV1-42, IGHV1-50, IGHV187 , and IGHV1S-45 showed significant differences with changes in age $(P<0.05)$. 
D gene usage (Fig. 2-2): (1) Productive, pseudogene, and out-of-frame sequence high-frequency usage genes were concentrated in IGHD1-1, IGHD2-1, IGHD2-3, and IGHD2-4. (2) Productive sequences: Spleen B cell IGHD2-5, IGHD3-3, and IGHD6-3 and spleen memory B cell IGHD2-3, IGHD3-1, IGHD3-2, and IGHD3-3 showed significant differences with age $(\mathrm{P}<0.05)$. (3) Pseudogene sequences: Bone marrow $\mathrm{B}$ cell IGHD23, and IGHD2-4 and spleen B cell IGHD3-1, IGHD3-3, and IGHD5-1 showed significant differences with changes in age $(P<0.05)$. (4) Out-of-frame sequences: The frequency of spleen $B$ cell and memory $B$ cell IGHD2-4 in 20-month-old mice was greater than that in 12- and 3-month old mice; bone marrow $\mathrm{B}$ cell IGHD2-2, IGHD2-4, IGHD3-2, and IGHD5-1, spleen B cell IGHD2-5, and spleen memory B cell IGHD1-3, IGHD2-1, IGHD2-12, IGHD2-5, IGHD3-1, IGHD3-2, IGHD4-1, and IGHD6-2 showed significant differences with changes in age $(P<0.05)$.

$J$ gene usage (Fig. 2-3): Productive sequences: The spleen B cell IGHJ2 gene showed significant differences with changes in age $(\mathrm{P}<0.05)$. Pseudogene sequences: The spleen B cell IGHJ1 gene showed significant differences with changes in age $(P<0.05)$.

$V-J$ pairing in the BCR H-CDR3 repertoire (Fig. S3-1, Fig. S3-2 and Fig. S3-2): In the productive, pseudogene, and out-of-frame sequences, the $V-J$ advantage pairing usage was performed differently. Cluster analysis showed that the bone marrow B cells, spleen B cells, and spleen memory B cells in the productive sequences had closer clustering distances at 12 months and 20 months. The pseudogene sequences had closer clustering of the bone marrow and spleen B cells at 3 months and 12 months, while spleen memory B cells were closer clustered at 12 months and 20 months. Out-of-frame sequences had closer clustering of bone marrow $B$ cells and spleen memory $B$ cells at 3 months and 12 months of age, while spleen B cells were closer clustered at 12 months and 20 months.

\section{Insertions and deletions of nucleotides in the BCR H-CDR3 repertoire}

The CDR3 diversity results from the " $N$ " nucleotides at the $\mathrm{V} \rightarrow \mathrm{D}(\mathrm{N} 1)$ and $\mathrm{D} \rightarrow \mathrm{J}(\mathrm{N} 2)$ junctions, exonuclease trimming ( $3^{\prime} \mathrm{V}$ trimmed, $5^{\prime} \mathrm{D}$ trimmed and $5^{\prime} \mathrm{J}$ trimmed) and the addition of palindromic " $\mathrm{P}$ " nucleotides (P3'V, P5'D and P5'J)[17]. According to the classification method of nucleotide insertion and deletion reported by Murugan et al[18], it was found that in the productive, pseudogene, and out-of-frame sequences, bone marrow $B$ cells had significant differences in 5 'J trimming with age $(P<0.05)$; spleen $B$ cell and memory B cell N1 insertion, N2 insertion, $\mathrm{P} 5^{\prime} \mathrm{D}$ insertion, and $5^{\prime} \mathrm{D}$ trimming were significantly different $(P<0.05)$, (Fig. 3).

\section{Productive sequence clone proliferation in the BCR H-CDR3 repertoire}

An analysis of productive sequence clone proliferation was conducted using the inverse of Simpson's diversity index (1/DS), calculated as $1 / D S=1 / \sum\{n i *(n i-1)\} /\{n *(n-1)\}$, where ni refers to the total number of the ith sequence[19,20]. The higher the 1/DS value, the richer the diversity and the lower the clonal proliferation[21]. The frequencies of the unique sequences are ordered from high to low, and a log10 scale is used on the Y-axis to reveal the frequency (Fig. S4). The BCR H-CDR3 repertoire of mouse bone marrow B cells, spleen B cells and spleen memory B cells decreased with the increase in the 1/DS index 
(Fig. 4A ). In the BCR H-CDR3 repertoire of mouse spleen B cells, the 1/DS index at 12 months and 20 months was significantly lower than that at 3 months $(P<0.05)$ (Fig. 4B). The spleen memory $B$ cell $B C R$ H-CDR3 repertoire 1/DS index of 3-month-old mice was greater than that of 12-and 20-month-old mice (Fig. 4C).

\section{CDR3 length and amino acid usage in productive sequences}

The distribution of CDR3 lengths at different ages and in different tissues was similar and showed a Gaussian distribution(Fig. 5A, B, C). There was a statistically significant difference in the use of asparagine $(N)$ and isoleucine $(I)$ in the spleen $B$ cell repertoire with increasing age $(P<0.05)$. There was a statistically significant difference in the use of asparagine $(\mathrm{N})$ in the spleen memory $B$ cell repertoire with increasing age $(P<0.05)$, (Fig. 5D, E, F).

\section{Overlap in the productive sequences of the CDR3 repertoire}

The ratio of overlap to the number of unique amino acids in the mice at different ages was calculated. The proportion of overlap in bone marrow and spleen B cells in 3-month old mice was lower than that in 12- and 20-month-old mice. This observation was not seen in spleen memory B cells (Fig. 6, Tab. 2-1, 2-2, 2-3).

\section{Discussion}

As the age of mice increases, the thymus and spleen T cells TCR CDR3 repertoire will change [22, 23], at the same time, the number and response capacity of central and peripheral B cells will change accordingly. The homogeneity and heterogeneity of the composition and characteristics of the central and peripheral B cells in younger/middle/old-aged mice is an important basis for the study of the responsive ability and mechanism of the aging immune system. In this experiment, 3-, 12-, and 20-monthold mice were used as subjects, and HTS was used to compare and analyze the homogeneity and heterogeneity of the productive, pseudogene, and out-of-frame sequences of the BCR H-CDR3 repertoire in bone marrow (central) and spleen (peripheral).

The study found that the white pulp of the spleen was more regular at 3 months of age, the density of lymphocytes was larger, and the shape of the shifting area is obvious. With the increase in the age of the mice, the structure of the white pulp became irregular at the ages of 12 months and 20 months. Some of them are relatively loose, with only a small number of lymphocytes, and the shape of the area became irregular. The white pulp structure is typically associated with antigenic stimulation; as the mouse age increased, the mice received more antigenic stimulation, resulting in an irregular white pulp structure in aged mice, which is consistent with previous literature reports[24].

The diversity of the BCR H-CDR3 repertoire is derived from the $V(D) J$ gene rearrangement, insertion, deletion, and high-frequency mutations in somatic cells. As mice age, changes in BCR H-CDR3 repertoire diversity can be demonstrated by random combinations of light and heavy 
chains[25]. The frequency of the $V, D$, and $J$ genes was related to the advantages of naive rearrangement, $B$ cell self-tolerance selection, $B$ cell clonal proliferation, immune response, etc. In theory, the frequency of the usage of the $V, D$, and $J$ genes reflects the key features of the CDR3 recognition of specific antigens. This study found a high frequency of the usage of IGHV1-4, IGHV14-1, IGHV1-50, and IGHV1-64 in the productive and out-of-frame sequences of bone marrow B cells, spleen B cells and spleen memory B cells at different ages. In the pseudogene sequences, the frequency of IGHV1-67 and IGHV1-83 was significantly higher than that of other genes; IGHV1 had high-frequency usage at different ages and in different tissues, showing homogeneity, which is consistent with other researchers' reports[8]. This finding suggests that in the naive rearrangement, there is a significant advantage of some $V$ gene in the naive rearrangement, resulting in its high frequency in the self-tolerance selection and peripheral response BCR repertoire. In the productive sequences, mouse bone marrow B cell IGHV1-54 and IGHV1-84; spleen B cell IGHV4-1, IGHV1-87, IGHV5-12, and IGHV9-1; and spleen memory B cell IGHV1-4, IGHV5-12-1, and IGHV5-9 usage significantly changed with age. Significant changes in gene usage may be related to $B$ cell tolerance, clonal proliferation, and the frequency of the immune response and further indicate that the BCR H-CDR3 repertoire is partially heterogeneous at different ages and in different tissues. In the analysis of the frequency of IGHV gene family use, it was found that some IGHV gene families did not show regular changes with the increase of months ( 3 months, 12 months, and 20 months). This may require a more detailed analysis of the $B$ cell subsets sorted in the bone marrow and spleen. The experiment needs to further clarify the characteristics of the naïve B cells and memory B cells (and different types of memory $B$ and subsets) CDR3 repertoire in different parts of the bone marrow or spleen (Such as IGHV gene family utilization frequency) changes dynamically with the age of the month.

A pseudogene sequence is produced by the deletion of the initiation codon and the premature introduction of a termination codon[9]. A pseudogene sequence also participates in rearrangement. Because of the inefficiency of rearrangement, the pseudogene sequence better reflects the frequency characteristics of the $V, D$ and $J$ genes in the naive rearrangement, and, together with the out-of-frame rearrangement of functional genes, it can be used to compare and analyze the composition and specificity of the productive rearrangement of functional genes[26].

The pseudogene sequence showed significant differences in bone marrow B cell IGHV1-25, IGHV1-83, IGHD2-3 and IGHD2-4 with age and significant differences in spleen B cell IGHV1-32, IGHV1-67, IGHD3-1, IGHD3-3, and IGHD5-1 genes. Compared with the productive sequences, there were differences in $V$ and $D$ gene usage, suggesting that the pseudogene sequence better reflects naive rearrangement. A out-offrame sequence is the result of non-productive rearrangementwe. When we analyzed the $V$ genes usage, we found that the out-of-frame sequence and the Productive sequence have many similarities, mainly because the out-of-frame sequence sequence is a translational reading frame change caused by insertion and deletion of nucleotides during gene rearrangement and cannot be translated into functionality AA. Therefore, we believe that the difference in the out-of-frame sequence in the bone marrow tissue with age changes is mainly affected by gene rearrangement, or it may be the cause of individual differences. Therefore, we believe that the difference in the age of the out-of-frame sequence in the central and peripheral tissues is mainly affected by gene rearrangement, or it may be affected by the antigen 
response in the periphery.The frequency of the acquisition of the $V, D$, and $J$ genes in mice was almost the same, and there were some differences with changes in mouse age, which is consistent with the study of the $V, D$, and $J$ genes in the peripheral blood of newborns and adults[27].

The closer the cluster analysis is, the more similar the gene access is. The cluster analysis of $V-J$ pairing in spleen B cells and memory B cells shows that the clustering distances of 12 months and 20 months are the closest, while the cluster distance of 3 months is more. Indicating that the 12-month-old and 20month-old genes are more similar, suggesting that it may be similar to the longer-term in vitro antigen stimulation, and $\mathrm{B}$ cells produce corresponding responses and clonal proliferation. The clustering distance of pseudogene sequences was closer at 3 months and 12 months than at 20 months in the bone marrow and spleen B cells. In the out-of-frame sequences in bone marrow B cells and spleen memory B cells at the ages of 3 months and 12 months, the clustering distance was closer than that at 20 months. It is suggested that more $V$ and $J$ gene naïve rearrangements have higher advantages and matching. The specific sources and mechanisms of various $V$-J pairing B cell populations need to explore its regular changes in experiments with more mouse-month-old groups.

For the insertions and deletions of the BCR H-CDR repertoire, in the productive, pseudogene, and out-offrame sequences, the bone marrow $B$ cells had significant differences in $5^{\prime} \mathrm{J}$ trimming with age and may be mainly pro-B cells and pre-B cells.In the pro-B cell repertoire, the rearrangement is generally random, but there is a certain difference in the involvement of the $J$ gene in functional and nonfunctional rearrangements. Therefore, in the next experiment, it is necessary to sort the pro-B cells and pre-B cells, naïve B cells and memory B cells in the bone marrow of mice of different months, and compare and analyze their characteristics in mice of different months. To explore the mechanism and significance of changes in central B cell rearrangement, selection and differentiation in the process of mice increasing with age. There were significant differences in spleen B cell and memory B cell N1 insertion, N2 insertion, $P 5 ' D$ insertion, and 5'D trimming, suggesting that the BCR H-CDR3 repertoire and B cell self-tolerance selection, immune response, and clonal proliferation are related. Due to the response of multiple autoantibodies and external antigens, the CDR3 repertoire participates in this process in addition to the naive rearrangement, high-frequency mutations of somatic cells and the secondary rearrangement of the $B C R$, resulting in N1 insertion, N2 insertion, $P 5^{\prime} D$ insertion, and 5'D trimming differences. Therefore, in the peripheral immune organs, it is more complicated to explore the mechanism and significance of insertion and deletion of the CDR3 repertoire of different subsets of B cells in the process of changing with the age of mice. It needs to be carried out on the basis of mouse disease models of different months.

The diversity of the BCR H-CDR3 repertoire in different ages of mice showed that the diversity in bone marrow B cells and spleen memory B cells of 3-month-old mice was higher than that of 12-and 20-month old mice. In spleen B cells, the diversity of the 3-month-old mouse repertoire was significantly higher than that of the 12-and 20-month old mouse repertoire. The diversity of the BCR H-CDR3 repertoire is closely related to gene rearrangement and the immune response generated by external antigen stimulation. The diversity of the repertoire in the bone marrow is mainly due to the rearrangement/insertion/deletion of the $V, D$, and $J$ genes[28]. As the mouse age increases, the $B$ cell output from the bone marrow to the 
periphery decreases, and the diversity decreases[5]. The findings may have occurred in the bone marrow, and this reduction may also reflect changes in the subset[20,25].

The response of spleen B cells to external antigen stimulation, as the body ages to increase the body's depletion of B cells, also leads to a decrease in B cell diversity, and B cell clonal proliferation changes with increasing age. Not only are the unique CDR3 sequence species reduced, but the clonal proliferation that occurs is also greater. This phenomenon also indicates that the clonal distribution caused by the steady-state proliferation and peripheral selection in the aging process is more unbalanced. Related studies indicate that the imbalance of clonal distribution can reflect the response ability and the peripheral selection of self-identification[29]. he changes in the diversity of the central and peripheral BCR $\mathrm{H}-\mathrm{CDR} 3$ repertoire in mice at 3,12 , and 20 months of age suggest that they are due to both central and peripheral causes, providing further insight into immune system aging and results from diversity studies, basic data and new research techniques.

The AA composition of the BCR H-CDR3 region was found to be high-frequency tyrosine in the B cells of different ages and tissue-derived $B$ cells, consistent with the literature[30]. The use of isoleucine $(I)$ in the spleen B cell repertoire was significantly higher at 12 months than at 3 months and 20 months, and the use of asparagine $(\mathrm{N})$ was significantly lower at 20 months than at 12 months. In the spleen memory $B$ cell repertoire, the frequency of asparagine $(\mathrm{N})$ was significantly lower at 3 months and 20 months than at 12 months. It is suggested that the difference in AA in the peripheral BCR repertoire of mice at different ages may be related to the immune response generated by specific antigen stimulation.

The length distribution analysis of CDR3 revealed that the length of the bone marrow $B$ cells, spleen $B$ cells and spleen memory B cells of mice at different ages showed a Gaussian distribution with 11-12 AA residues. This is consistent with the result previously reported for mouse CDR3, which was an average length of $11.5 \pm 1.9$ AA residues[17]. Compared with 3 months of age, the CDR3 length distribution in the BCR H-CDR3 repertoire of bone marrow $B$ cells, spleen B cells, and spleen memory B cells shifted to the left at 12 months and shifted to the right at 20 months, suggesting that with the increase in age, recognition antigen-producing responses, mutations, and clonal proliferation have a tendency to become longer and may be related to the antigens that are exposed[20]. Pickman Y., et al. also found healthy elderly people's BCR CDR3 length distributions can be distinguished from those of the young[31].

The overlap rate analysis of the B cell CDR3 repertoire is closely related to VDJ gene recombination selection, self-tolerance selection, and environmental immune response. This study found that the overlap ratio of the B cell CDR3 repertoire of mice of different months is higher (Fig. 6 and Tab. 2). The specific mechanism needs to be further explored, but through comparative analysis, it is found that the proportion of overlap in the bone marrow B cells and spleen B cells of mice at different ages was lower at 3 months and higher at 12 months and 20 months. The overlap of unique amino acids at different ages reflects not only clonal proliferation but also the presence of new, effective, unique sequences. Our results showed that the overlap rate of bone marrow and spleen B cells at 3 months was lower, and the overlap rate at 12 and 20 months was higher, which suggests that clonal proliferation increases with age. 
Aranburu A., et al. found age-associated B cells (ABCs) in autoimmune mice are comprised of autoreactive MBCs expressing highly restricted $\mathrm{H}-\mathrm{CDR} 3$ repertoires[32]. In our study, the spleen memory $\mathrm{B}$ cells have no obvious regularity, which is mainly affected by the stimulation of the external antigen response.

Although this study found that the CDR3 repertoire of bone marrow and spleen B cells and spleen memory B cells in mice have multiple heterogeneity and other characteristics with the change of months, However, it is necessary to further clarify the relationship between naïve B cells

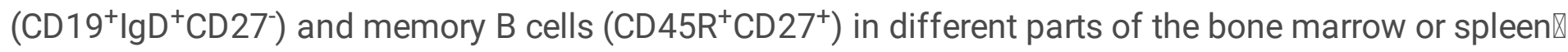
and even the memory $\mathrm{B}$ cells $\left(\mathrm{CD} 45 \mathrm{R}^{+} \mathrm{CD} 27^{+}\right)$in the bone marrow and spleen need to be further sorting by the method, conduct research on the CDR3 repertoire, such as comparing the CDR3 repertoire of T-bet and T-bet $^{+}$memory $B$ cells in the spleen germinal center or circulation with age[33]. Exploring the characteristics of B cell BCR in mice of different months of age can provide a basis for clinical disease mechanism research. For example, Zhang J., et al. found that B cell BCR activation is different in similar diseases of different ages, adult AML samples have significantly higher level of $B$ cell activation and more secondary Ig class switch events than pediatric AML or non-tumor samples[34, 35].

\section{Conclusion}

The degradation of the immune system related to aging is a dynamic process that affects the diversity and response capacity of the body's immune repertoire. In this study, HTS was used to investigate the effects of aging on the characteristics of the BCR H-CDR3 repertoire in mouse bone marrow $B$ cells, spleen B cells and spleen memory B cells. We found that the productive, pseudogene, and out-of-frame sequences of bone marrow B cells, spleen B cells and spleen memory B cells in 3-, 12-, and 20-month-old mice have different compositions, and some features show significant heterogeneity, which further provides a basis for investigating the decline and response of B cell immunity in younger/middleaged/older mice.

\section{Methods}

\section{Study subjects}

Three-month-old, 12-month-old, and 20-month-old SPF female BALB/c mice were purchased from Chongqing Tengxin Biotechnology Co., Ltd., and introduced into the Central Laboratory Animal Center of Zunyi Medical University (SPF feeding conditions). All animals and experiments were performed in accordance with the guidelines of the Animal Care and Use of Laboratory Animals (Ministry of Health, China, 1998) and approved by the Laboratory Animal Ethics Committee of Zunyi Medical University.

\section{Sample preparation}


(1) Mouse bone marrow $B$ cell samples (5 mice per month): 3 months old (M3BM): M3BM1, M3BM2, M3BM3, M3BM4, M3BM5; 12 months old (M12BM): M12BM1, M12BM2, M12BM3, M12BM4, M12BM5; and 20 months old (M20BM): M20BM1, M20BM2, M20BM3, M20BM4, M20BM5. (2) Mouse spleen B cell samples (5 mice per month): 3 months old (M3S): M3S1, M3S2, M3S3, M3S4, M3S5; 12 months old (M12S): M12S1, M12S2, M12S3, M12S4, M12S5; and 20 months old (M20S): M20S1, M20S2, M20S3,

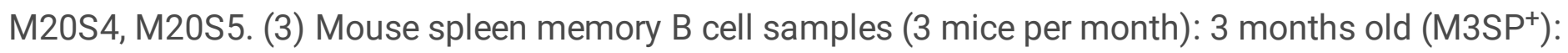
M3S7, M3S8, M3S9; 12 months old (M12SP $)$ : M12S7, M12S8, M12S9; and 20 months old (M20SP+): M20S7, M20S8, M20S9.

\section{Preparation of single-cell suspension}

Mice were dissected to remove the spleen and bone marrow for tissue sectioning. A single-cell suspension of memory B cells $\left(C D 45 \mathrm{R}^{+} \mathrm{CD} 27^{+}\right)$was prepared with Miltenyi bead sorting, and the purity of the sorted cells was identified. The medullary cavity was washed of the bone marrow cells with PBS to prepare a single-cell suspension, and then, the genomic DNA in the bone marrow and in the spleen singlecell suspension of the memory B cell sample was extracted.

\section{BCR H-CDR3 sequencing}

This experiment is based on the gene composition of 16 large families of mouse functional IGHV, pseudogene IGHV and ORF IGHV, and 4 families of mouse IGHJ, we designed and synthesis of 16 upstream primers and 4 downstream primers of the mouse, as well as 2 GAPDH primers, were conducted by Shanghai Invitrogen Biotechnology Co., Ltd. (Tab. S1-1; S1-2; S1-3).

The DNA was used as a template to PCR amplify the BCR H-CDR3 region, with 100-200 bp PCR product agarose gel recovery (Fig. S1), and the HTS of the CDR3 repertoire was completed by BGI.

\section{Data analysis}

The CDR3 data of each mouse at each month's age were analyzed separately for composition characteristics. The CDR3 sequence of the HTS was converted into FASTA format and uploaded to the IMGT database (Use IMGT/HighV-QUEST tool and IMGT/GENE-DB database to provide total MUS data for analysis). The sequences with no results, unknown or $<90 \% \mathrm{~V} / \mathrm{J}$-region identity, AA junctions that did not have a $\mathrm{C}$ at the beginning or a $\mathrm{W}$ at the end, fewer than 6 nucleotides, blanks and ORFs were filtered out from the data downloaded by IMGT. The selected productive, pseudogene, and out-of-frame sequences were statistically analyzed using Excel, GraphPad Prism, Helm, SPSS (one-way ANOVA), and Draw Venn Diagram online software. For statistical significance, * indicates $P<0.05$, ** indicates $P<0.01$, and $* \star *$ indicates $P<0.001$.

\section{Abbreviations}


1/DS: The inverse Simpson'sdiversity index; AA: Amino Acid; BCR: B Cell Receptor; SPF: Specific Pathogen Free; H-CDR3: Heavy chain Complementarity Determining Region 3; HTS: High-Throughput Sequencing; IGHV: Immunoglobulin Heavy chain Variable gene; IGHD: Immunoglobulin Heavy chain Diversity gene; IGHJ: Immunoglobulin Heavy chain Jointing gene; ORF: Open Reading Frame; C: Cysteine; W: Tryptophan.

\section{Declarations}

\section{Acknowledgements}

We are grateful to all for supporting this study, and we thank all the authors, GBI, IMGT for using their HTS data for analysis.

\section{Authors contributions}

Xinsheng Yao designed the research. Lina Ma did the experiment, analyzed the data and wrote the paper. Xinxin Tao, Xiaoyan He, Peng wang, Long Ma, Bin Shi, participated in experiments and data analysis. All authors read and approved the final manuscript.

\section{Funding}

The work was supported by grants from the National Natural Science Foundation of China (81860300\&81660269) and Guizhou Provincial High-level Innovative Talents Project (No. [2018] 5637)

\section{Availability of data and materials}

The datasets used and/or analyzed during the current study are available from the corresponding author on reasonable request.

\section{Ethics approval and consent to participate}

All animals and experiments were performed in accordance with the guidelines of the Animal Care and Use of Laboratory Animals (Ministry of Health, China, 1998) and approved by the Laboratory Animal Ethics Committee of Zunyi Medical University.

\section{Consent for publication}

Written consent for publication was obtained from Zunyi Medical University.

\section{Competing interests}

The authors declare that they have no competing interests.

\section{References}


1. Frasca, D., et al., Age effects on B cells and humoral immunity in humans. Ageing Res Rev, 2011.10(3): p. 330-5.

2. Frasca, D., et al., Aging down-regulates the transcription factor E2A, activation-induced cytidine deaminase, and Ig class switch in human B cells. J Immunol, 2008. 180(8): p. 5283-90.

3. Cong X; Zhao Q, Immune system and body aging. Chinese Journal of Aesthetic Medicine, 2017. 26: p. $16-20$.

4. Bulati, M., et al., B cells and immunosenescence: a focus on IgG+lgD-CD27- (DN) B cells in aged humans. Ageing Res Rev, 2011. 10(2): p. 274-84.

5. Gibson, K.L., et al., B-cell diversity decreases in old age and is correlated with poor health status. Aging Cell, 2009. 8(1): p. 18-25.

6. Dunn-Walters, D.K., The ageing human B cell repertoire: a failure of selection? Clin Exp Immunol, 2016. 183(1): p. 50-6.

7. The diversity of the naive BCR H-CDR3 repertoire is derived from the rearrangement of the germline $\mathrm{V}(\mathrm{D}) \mathrm{J}$ gene in the bone marrow

8. Schroeder, H.W., Jr., Similarity and divergence in the development and expression of the mouse and human antibody repertoires. Dev Comp Immunol, 2006. 30(1-2): p. 119-35.

9. Roberts, T.C. and K.V. Morris, Not so pseudo anymore: pseudogenes as therapeutic targets. Pharmacogenomics, 2013. 14(16): p. 2023-34.

10. Calame, K.L., Plasma cells finding new light at the end of B cell developm nt.pdf. Nature Immunology, 2001. 12: p. 1103-1109.

11. Dunn-Walters, D.K. and A.A. Ademokun, B cell repertoire and ageing. Curr Opin Immunol, 2010. 22(4): p. 514-20.

12. Caruso, C., et al., Mechanisms of immunosenescence. Immun Ageing, 2009. 6: p. 10.

13. Monroe, J.G. and K. Dorshkind, Fate Decisions Regulating Bone Marrow and Peripheral B Lymphocyte Development. 2007. p. 1-50.

14. Cancro, M.P., et al., B cells and aging: molecules and mechanisms. Trends Immunol, 2009. 30(7): p. 313-8.

15. Aberle, J.H., et al., Mechanistic insights into the impairment of memory B cells and antibody production in the elderly. Age (Dordr), 2013. 35(2): p. 371-81.

16. Siegrist, C.A. and R. Aspinall, B-cell responses to vaccination at the extremes of age. Nat Rev Immunol, 2009. 9(3): p. 185-94.

17. Shi, B., et al., Compositional characteristics of human peripheral TRBV pseudogene rearrangements. Sci Rep, 2018. 8(1): p. 5926.

18. Murugan, A., et al., Statistical inference of the generation probability of T-cell receptors from sequence repertoires. Proc Natl Acad Sci U S A, 2012. 109(40): p. 16161-6.

19. van Heijst JW, C.I., Lipuma L B, et al. , Quantitative assessment of T cell repertoire recovery after hematopoietic stem cell transplantation.pdf. Nature Medicine, 2013. 19: p. 372-378. 
20. Wang, C., et al., Effects of aging, cytomegalovirus infection, and EBV infection on human B cell repertoires. J Immunol, 2014. 192(2): p. 603-11.

21. Wu, J., et al., T-cell receptor diversity is selectively skewed in T-cell populations of patients with Wiskott-Aldrich syndrome. J Allergy Clin Immunol, 2015. 135(1): p. 209-16.

22. Li Y, Ma L, Dong X, Pan Y, Shi B, He X, Zhang T, Sun S, Yao X. Preliminary analysis of spatial-temporal homogeneity and heterogeneity of TCR $\beta$ chain CDR3 repertoires in BALB/c mice. Int J Mol Epidemiol Genet. 2019 Feb 15;10(1):10-28. eCollection 201.

23. Li Z, Long M, ChunMei L, Bin S, Jiang Y, Rui M, Qingqing M, XinSheng Y.Composition and variation analysis of TCR $\beta$-chain CDR3 repertoire in the thymus and spleen of MRL/Ipr mouse at different ages. Immunogenetics. 2015 Jan;67(1):25-37.

24. Feng R.; Pang $\mathrm{H} ; \mathrm{He} \mathrm{W}$, e., al. , Age-related change in the structure and function of the immune system in Balb/C mice. Chinese Journal of Geriatrics, 2000. 19: p. 174-178.

25. Scholz, J. L., et al., A comparative review of aging and B cell function in mice and humans. Curr Opin Immunol, 2013. 25(4): p. 504-10.

26. Niimura, Y. and M. Nei, Extensive gains and losses of olfactory receptor genes in mammalian evolution. PLoS One, 2007. 2(8): p. e708.

27. Hong, B., et al., In-Depth Analysis of Human Neonatal and Adult IgM Antibody Repertoires. Front Immunol, 2018. 9: p. 128.

28. Granato, A., Y. Chen, and D.R. Wesemann, Primary immunoglobulin repertoire development: time and space matter. Curr Opin Immunol, 2015. 33: p. 126-31.

29. McClellan, A.J., et al., Ocular surface disease and dacryoadenitis in aging C57BL/6 mice. Am J Pathol, 2014. 184(3): p. 631-43.

30. Shi B, M.L., He X, et, al., Comparative analysis of human and mouse immunoglobulin variable heavy regions from IMGTILIGM-DB with IMGTIHighV-QUEST. Theor Biol Med Model, 2014. Vol.11.

31. Pckman Y, Dunn-Walters D, Mehr R. BCR CDR3 length distributions differ between blood and spleen and between old and young patients, and TCR distributions can be used to detect myelodysplastic syndrome. Phys Biol. 2013 Oct;10(5):056001.

32. Aranburu A, Höök N, Gerasimcik N, , et, al. Age-associated B cells expanded in autoimmune mice are memory cells sharing H-CDR3-selected repertoires..Eur J Immunol. 2018 Mar;48(3):509-521

33. John L Johnson, Rebecca L Rosenthal, et, al. The Transcription Factor T-bet Resolves Memory B Cell Subsets with Distinct Tissue Distributions and Antibody Specificities in Mice and Humans.Immunity.2020 May 19;52(5):842-855.

34. Zhang J, Hu X, et, al. Immune receptor repertoires in pediatric and adult acute myeloid leukemia. Genome Med. 2019 Nov 26;11(1):73.

35. Davydov AN, Obraztsova AS, et, al. Comparative Analysis of B-Cell Receptor Repertoires Induced by Live Yellow Fever Vaccine in Young and Middle-Age Donors Front Immunol. 2018 Oct 9;9:2309. 


\section{Tables}

Table 1. The BCR H-CDR3 repertoire of productive, pseudogene, and out-of-frame sequences in mice of different ages 


\begin{tabular}{|c|c|c|c|}
\hline \multirow[t]{2}{*}{ Sample } & Productive & Pseudogene & Out of Frame \\
\hline & Total[unique『 & Total[unique[ & Total[unique® \\
\hline M3BM1 & 3662734ロ474483ם & $107165 \square 20484 \square$ & 350156ロ89830ם \\
\hline M3BM2 & 4201156ロ522977ロ & $128828 \square 21769 \square$ & $331242 \llbracket 87427 \square$ \\
\hline M3BM3 & $772633 \square 386000 \square$ & 16473ロ16473ロ & $137409 \square 75974 \square$ \\
\hline M3BM4 & 1357086ロ177848ロ & $50529 \llbracket 8549 \square$ & 305304₫54945■ \\
\hline M3BM5 & 1296498凸178251₫ & $42892 \square 8104 \square$ & $348632 \llbracket 61586 \square$ \\
\hline M12BM1 & 1438032₫197994₫ & 35419ロ6960ロ & 97826ロ30317ロ \\
\hline M12BM2 & 1477622₫156699』 & 58100ロ8877ロ & $231830 \square 45326 \square$ \\
\hline M12BM3 & 502958ロ64768ם & $16480 \square 2915 \square$ & $98908 \square 20059 \square$ \\
\hline M12BM4 & 1108553ロ184165ロ & $34074 \square 7926 \square$ & $207506 \llbracket 51285 \square$ \\
\hline M12BM5 & 1669642₫170188』 & $66465 \square 8764 \square$ & 324449₫50027₫ \\
\hline M20BM1 & 1494519ロ242290๘ & $39108 \square 9174 \square$ & 400643₫87675』 \\
\hline M20BM2 & 1316534ロ199635ロ & 45487ロ9149ロ & 277021₫58823ם \\
\hline M20BM3 & 561490ロ120788ロ & $19500 \square 5264 \square$ & $120708 \square 31740 \square$ \\
\hline M20BM4 & 429629ロ76188ロ & 11326ロ2883ロ & 78493ロ17853凸 \\
\hline M20BM5 & 313694₫67759ロ & $8452 \square 2542 \square$ & 48381ロ13366ロ \\
\hline M3S1 & 4523881ロ786508』 & 149238ロ33361₫ & 865160ロ208318\ \\
\hline M3S2 & 4017413๘682554ロ & $108054 \square 25438 \square$ & $663139 \llbracket 213543 \square$ \\
\hline M3S3 & $4417442 \square 637351 \square$ & 137097ロ28256ロ & $784674 \square 160134 \square$ \\
\hline M3S4 & 3942536ロ606347ロ & 97789ロ22957ロ & 808071ロ164970】 \\
\hline M3S5 & 2696776ロ571503ロ & 63029ロ18062ロ & 446298ロ122955ロ \\
\hline M3S7 & 1091791₫91001₫ & 44851₫4866凸 & $226582 \square 27110 \square$ \\
\hline M3S8 & 46895ロ12469ロ & 1165ロ446ロ & $6682 \llbracket 2261 \square$ \\
\hline M3S9 & 183534ロ27923ם & $5709 \square 1241 \square$ & $34871 \square 6784 \square$ \\
\hline M12S1 & 1044884ロ101677ロ & $15109 \square 3242 \square$ & $266668 \llbracket 20675 \square$ \\
\hline M12S2 & $1365544 \llbracket 280774 \square$ & 42482₫11209』 & $212575 \square 60657 \square$ \\
\hline M12S3 & 1382706ロ226375ロ & 28890ロ7327ロ & 217936ロ51437ロ \\
\hline M12S4 & 1555800ロ265748』 & $23832 \square 7127 \square$ & 329043₫70801』 \\
\hline M12S5 & 1441200ロ201908』 & $24061 \square 5619 \square$ & $267609 \llbracket 51811 \square$ \\
\hline M12S7 & 737198ロ137061₫ & 44046ロ7571ם & $57282 \square 21271 \square$ \\
\hline M12S8 & 880181ロ169410凸 & $14891 \square 4214 \square$ & $65870 \square 23320 \square$ \\
\hline M12S9 & 77364ロ11970ロ & 1056ロ330ロ & $4592 \square 1626 \square$ \\
\hline M20S1 & 1296983ロ134233ロ & $24433 \square 4355 \square$ & $564603 \llbracket 37880 \square$ \\
\hline M20S2 & 1646868ロ205593ロ & $43362 \square 8512 \square$ & $235117 \square 46844 \square$ \\
\hline M20S3 & $535873 \llbracket 125903 \square$ & $10757 \square 3624 \square$ & $100049 \llbracket 29217 \square$ \\
\hline M20S4 & $1000811 \square 172421 \square$ & $22525 \square 5906 \square$ & $246581 \square 49540 \square$ \\
\hline M20S5 & 579776ロ156417ロ & 16389ロ5813ロ & $116676 \square 36376 \square$ \\
\hline M20S7 & 197807ロ22819ロ & 5327ロ923ロ & 114176ロ6685ロ \\
\hline
\end{tabular}




\begin{tabular}{lrrr} 
M20S8 & $717148 \square 68896 \square$ & $18116 \square 2575 \square$ & $634418 \square 24260 \square$ \\
M20S9 & $55512 \square 6239 \square$ & $2232 \square 327 \square$ & $11120 \square 1928 \square$ \\
\hline
\end{tabular}

Table 2-1. Statistical table of unique AA sequence overlap in the CDR3 sequences of bone marrow B cells in mice at different ages

\begin{tabular}{cccc}
\hline Sample & M3BM & M12BM & M20BM \\
& Overlap(Overlap/Unique) & Overlap(Overlap/Unique) & Overlap(Overlap/Unique) \\
\hline M3BM & 1016234 & $76476(18.485 \%)$ & $68131(15.685 \%)$ \\
M12BM & $76476(7.525 \%)$ & 413723 & $42998(9.899 \%)$ \\
M20BM & $68131(6.704 \%)$ & $42998(10.393 \%)$ & 434374 \\
\hline
\end{tabular}

Table 2-2. Statistical table of unique AA sequence overlap in the CDR3 sequences of spleen B cells in mice at different ages

\begin{tabular}{cccc}
\hline Sample & M3S & M12S & M20S \\
& Overlap(Overlap/Unique) & Overlap(Overlap/Unique) & Overlap(Overlap/Unique) \\
\hline M3S & 1750816 & $139163(22.576 \%)$ & $90369(18.834 \%)$ \\
M12S & $139163(7.948 \%)$ & 616433 & $63819(13.301 \%)$ \\
M20S & $90369(5.162 \%)$ & $63819(10.353 \%)$ & 479821 \\
\hline
\end{tabular}

Table 2-3. Statistical table of unique AA sequence overlap in the CDR3 sequences of spleen memory B cells in mice at different ages

\begin{tabular}{cccc}
\hline Sample & M3SP+ & M12SP+ & M20SP+ \\
& Overlap(Overlap/Unique) & Overlap(Overlap/Unique) & Overlap(Overlap/Unique) \\
\hline M3SP+ & 76508 & $10920(5.069 \%)$ & $3750(6.007 \%)$ \\
M12SP+ & $10920(14.273 \%)$ & 215421 & $7219(11.563 \%)$ \\
M20SP+ & $3750(4.901 \%)$ & $7219(3.351 \%)$ & 62430 \\
\hline
\end{tabular}

\section{Figures}


A
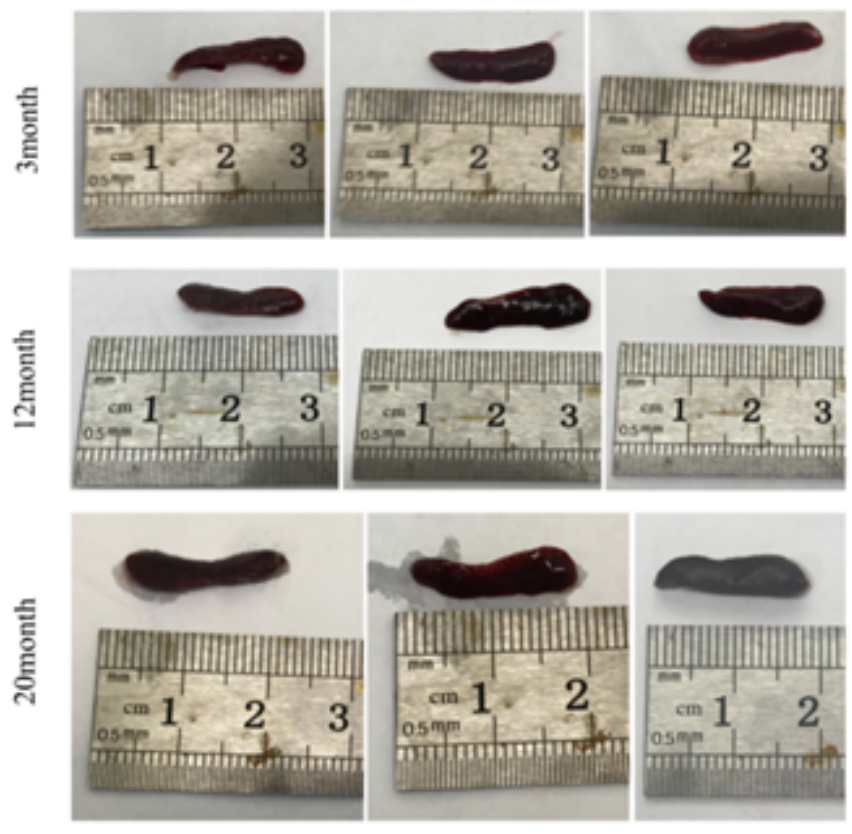

C
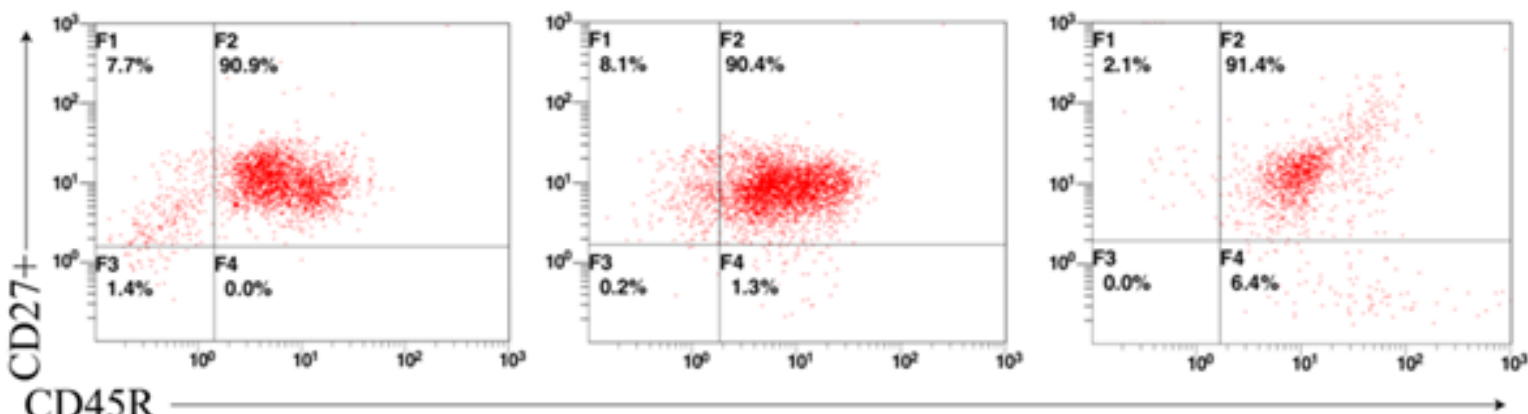

\section{Figure 1}

Preparation of mouse samples of different ages. A. Different ages of mouse spleen. B. HE stained sections of spleen tissues of mice at different ages (10X). C. Spleen memory B cell sorting purity identification. 
A
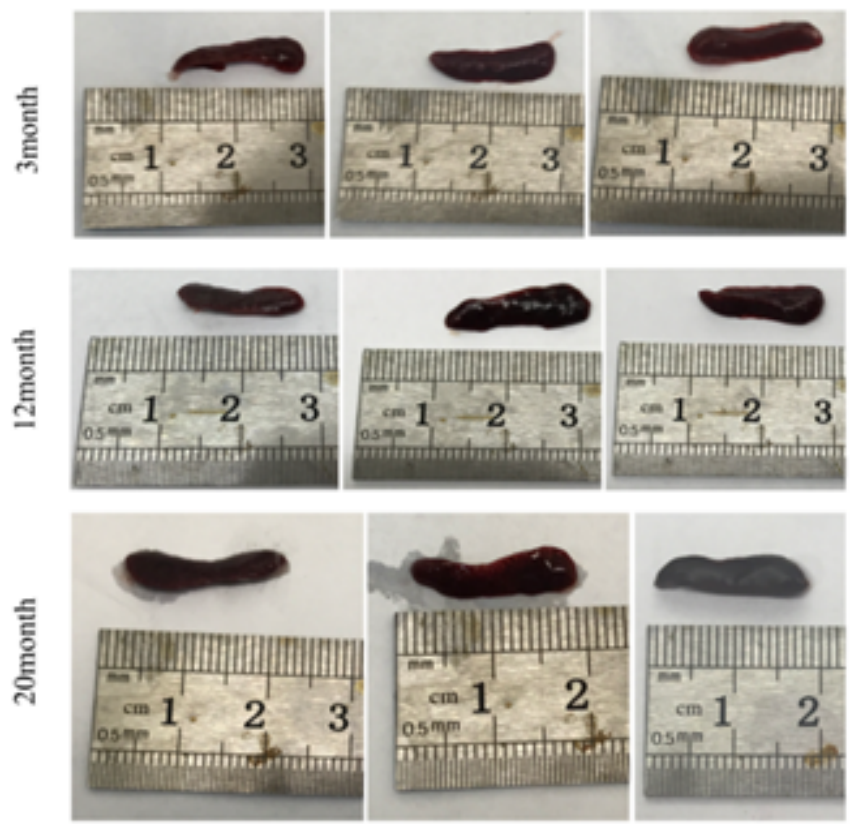

B
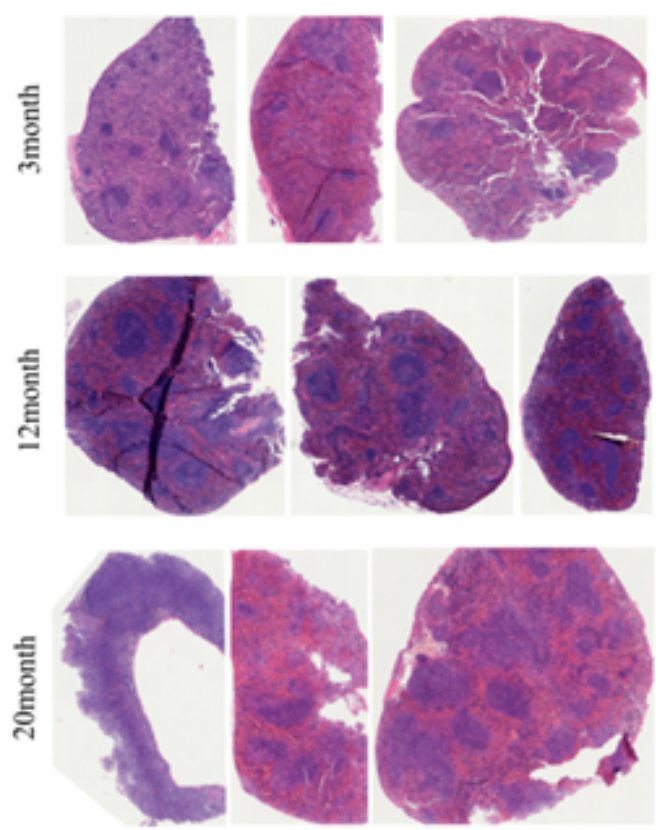

C
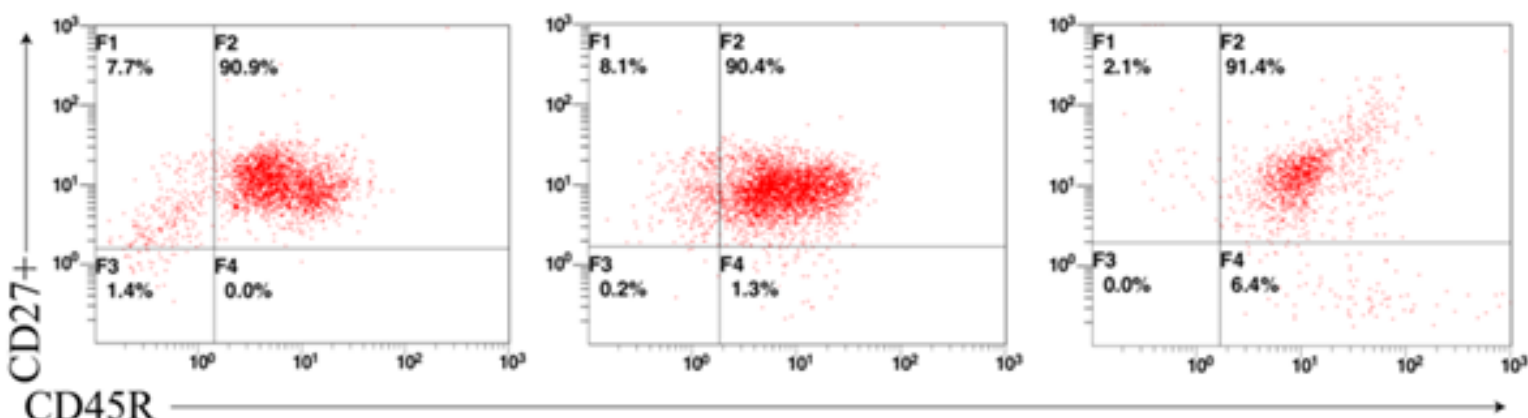

\section{Figure 1}

Preparation of mouse samples of different ages. A. Different ages of mouse spleen. B. HE stained sections of spleen tissues of mice at different ages (10X). C. Spleen memory B cell sorting purity identification.

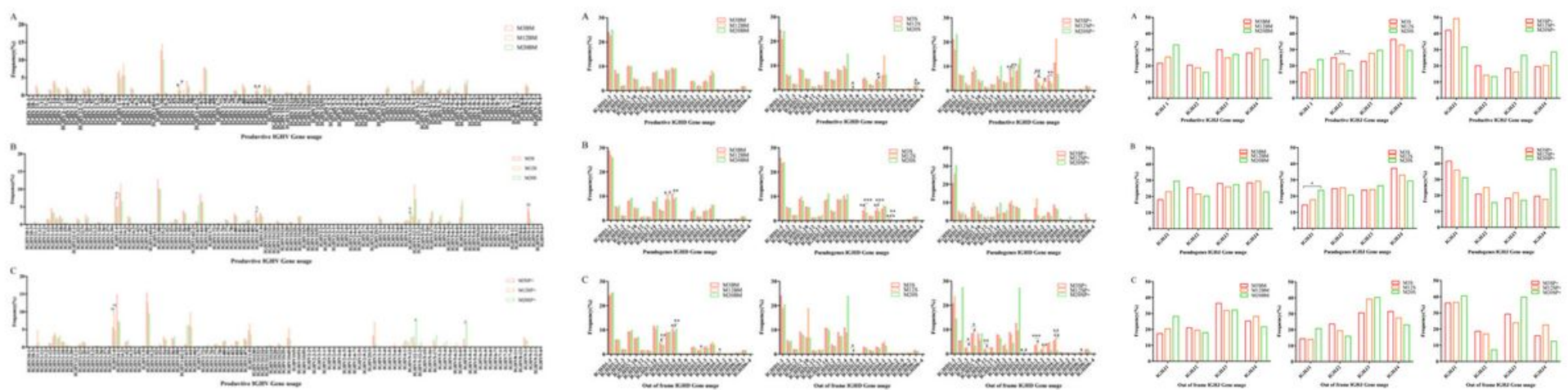

Figure 2 
(Left Panel) Productive sequence IGHV gene frequency in mice of different ages. A.The gene frequency of the IGHV gene in the bone marrow B cells. B.The gene frequency of the IGHV gene in the spleen B cells. C. The gene frequency of the IGHV gene in the spleen memory B cells. The $p$ values were determined using one-way ANOVA with a Bonferroni correction. All the statistically significant differences are indicated. $*=$ $p<0.05, * \star=p<0.01, * \star *=p<0.001$. (Middle Panel) The IGHD gene frequency in the mice of different ages. A. Productive sequence IGHD gene frequency.B. Pseudogene sequence IGHD gene frequency. C. Out-of-frame sequence IGHD gene frequency. The $p$ values were determined using one-way ANOVA with a Bonferroni correction. All the statistically significant differences are indicated. ${ }^{*}=p<0.05,{ }^{*}=p<0.01$, $\star \star \star ~=p<0.001$. (Right Panel) The IGHJ gene frequency in the mice of different ages. A. Productive sequence IGHJ gene frequency. B. Pseudogene sequence IGHJ gene frequency. C. Out-of-frame sequence IGHJ gene frequency. The $p$ values were determined using one-way ANOVA with a Bonferroni correction. All the statistically significant differences are indicated. $*=p<0.05, * *=p<0.01$
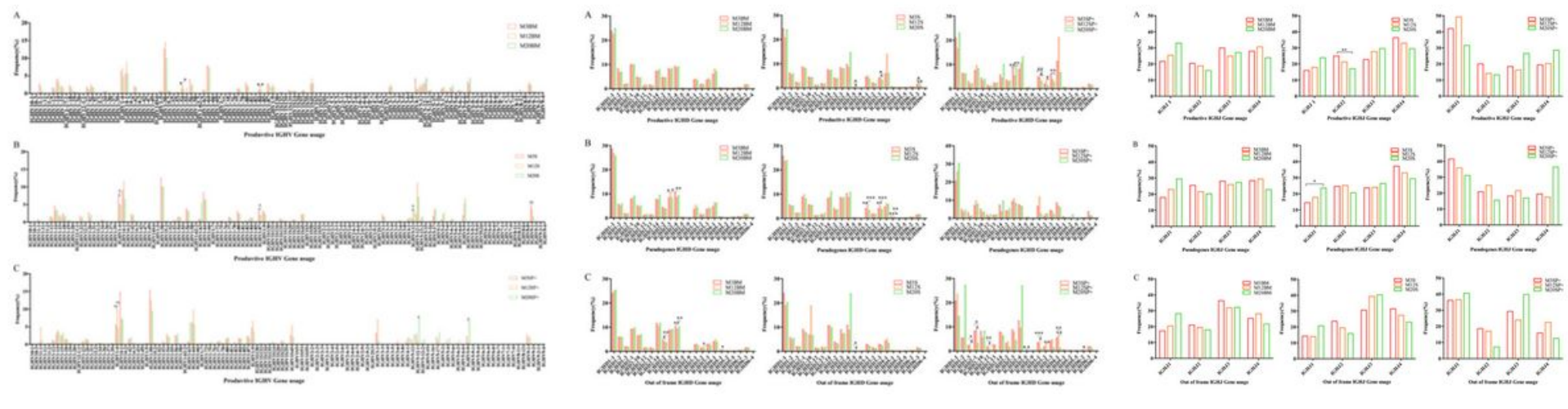

\section{Figure 2}

(Left Panel) Productive sequence IGHV gene frequency in mice of different ages. A.The gene frequency of the IGHV gene in the bone marrow B cells. B.The gene frequency of the IGHV gene in the spleen B cells. C. The gene frequency of the IGHV gene in the spleen memory B cells. The $p$ values were determined using one-way ANOVA with a Bonferroni correction. All the statistically significant differences are indicated. * $p<0.05,{ }^{* *}=p<0.01,{ }^{* \star}=p<0.001$. (Middle Panel) The IGHD gene frequency in the mice of different ages. A. Productive sequence IGHD gene frequency.B. Pseudogene sequence IGHD gene frequency. C. Out-of-frame sequence IGHD gene frequency. The $p$ values were determined using one-way ANOVA with a Bonferroni correction. All the statistically significant differences are indicated. ${ }^{*}=p<0.05, \star \star=p<0.01$, $\star \star \star ~=p<0.001$. (Right Panel) The IGHJ gene frequency in the mice of different ages. A. Productive sequence IGHJ gene frequency. B. Pseudogene sequence IGHJ gene frequency. C. Out-of-frame sequence IGHJ gene frequency. The $p$ values were determined using one-way ANOVA with a Bonferroni correction. All the statistically significant differences are indicated. ${ }^{*}=p<0.05,{ }^{* *}=p<0.01$ 

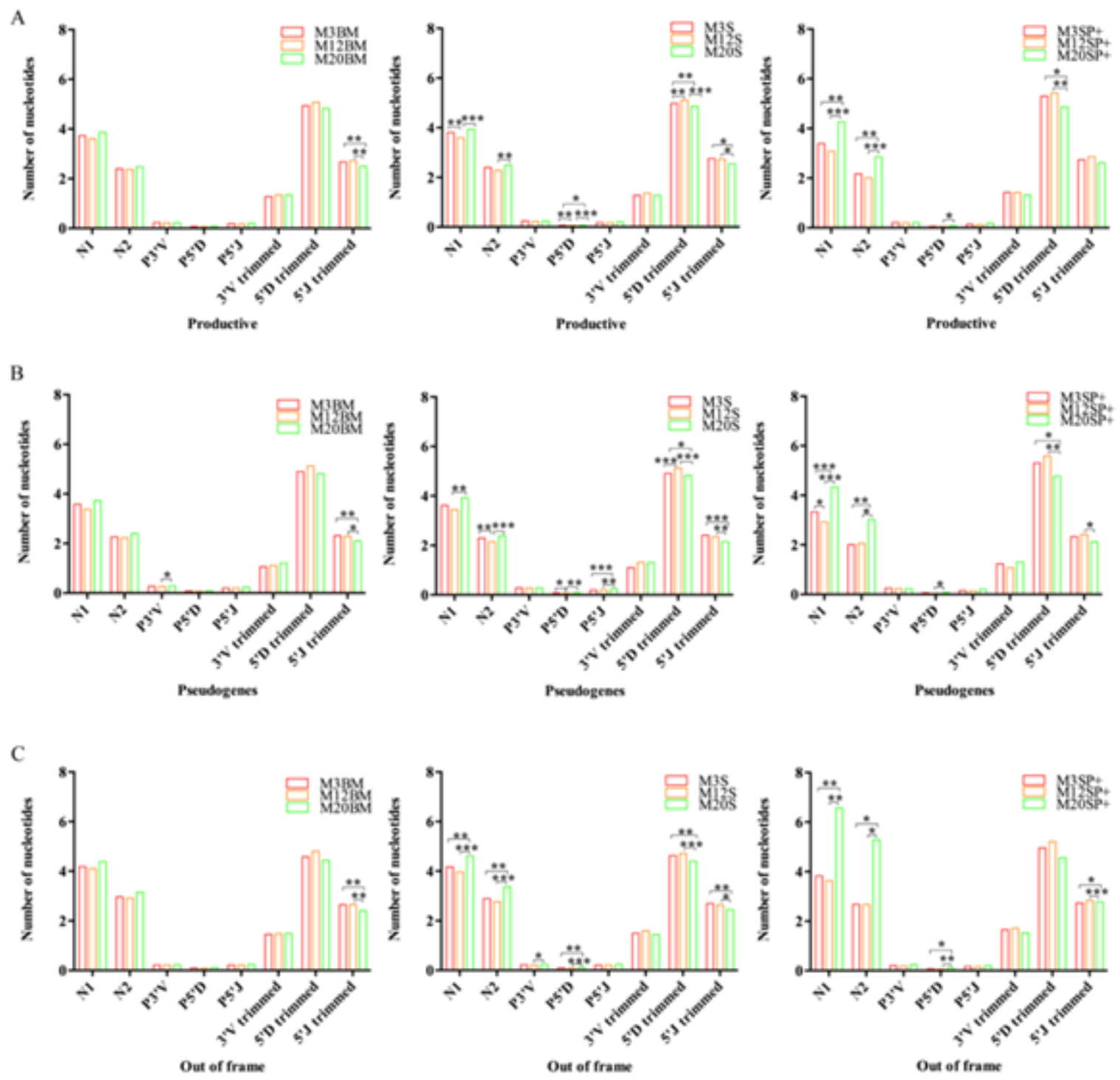

Figure 3

Insertion and deletion of nucleotides in the total sequences from mice of different ages. A. Productive sequences. B. Pseudogene sequences. C. Out-of-frame sequences. The number of N1, N2, P3'V, P5'D and P5'J nucleotides added at the V-D-J junctions and the nucleotides deleted at $3^{\prime} \mathrm{V}, 5^{\prime} \mathrm{D}$ and $5^{\prime} \mathrm{J}$ by exonuclease trimming are shown (mean \pm SD; error bars represent SD). The $p$ values were determined using one-way ANOVA with a Bonferroni correction. All the statistically significant differences are indicated. ${ }^{*}=p<0.05, * *=p<0.01, * \star *=p<0.001$. 

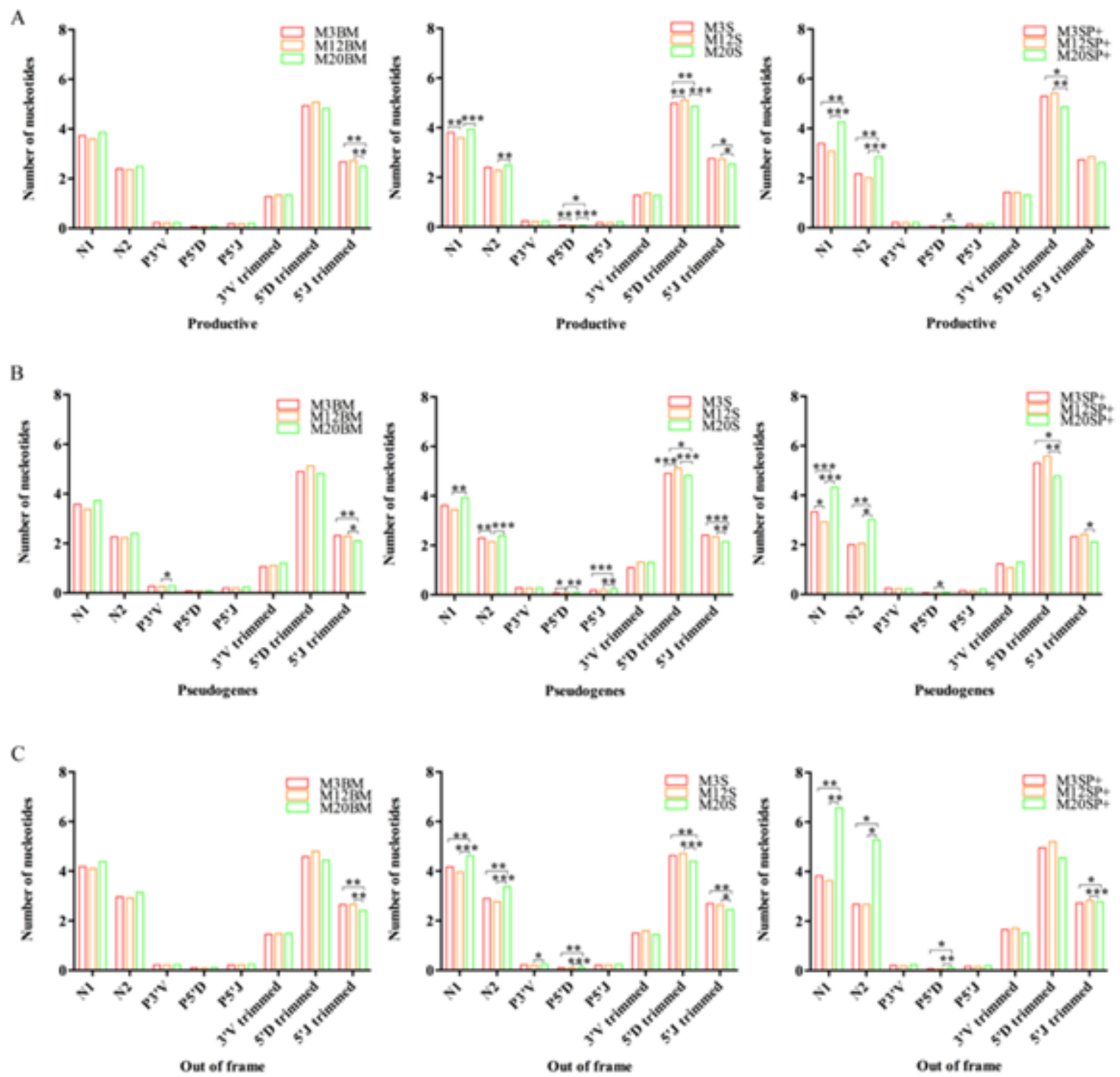

Figure 3

Insertion and deletion of nucleotides in the total sequences from mice of different ages. A. Productive sequences. B. Pseudogene sequences. C. Out-of-frame sequences. The number of N1, N2, P3'V, P5'D and P5'J nucleotides added at the V-D-J junctions and the nucleotides deleted at $3^{\prime} \mathrm{V}, 5^{\prime} \mathrm{D}$ and $5^{\prime} \mathrm{J}$ by exonuclease trimming are shown (mean \pm SD; error bars represent SD). The $p$ values were determined using one-way ANOVA with a Bonferroni correction. All the statistically significant differences are indicated. ${ }^{*}=p<0.05,{ }^{*}=p<0.01,{ }^{* *}=p<0.001$.

A

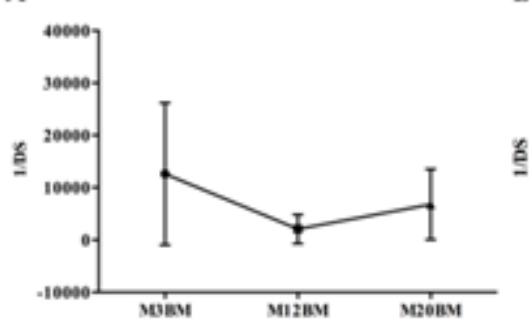

B

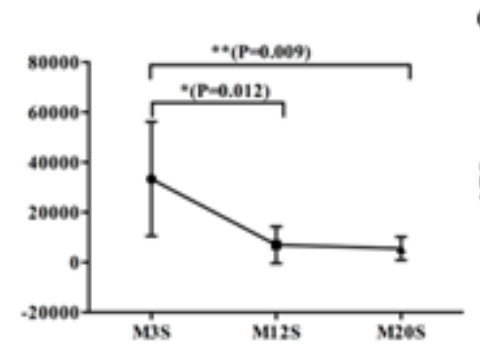

C

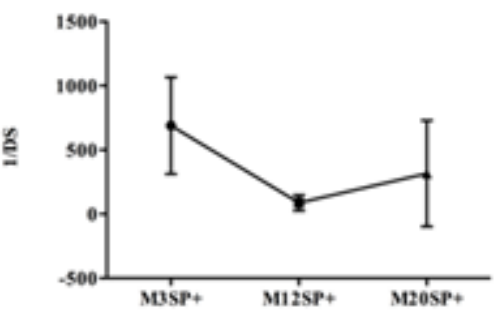




\section{Figure 4}

Diversity of the productive sequences of the BCR H-CDR3 repertoire in mice of different ages. A. Bone marrow B cell repertoire diversity. B. Spleen B cell repertoire diversity. C. Spleen memory B cell repertoire diversity. 1/DS are shown (mean $\pm S D$; error bars represent SD). The $p$ values were determined using oneway ANOVA with a Bonferroni correction. All the statistically significant differences are indicated. ${ }^{*}=\mathrm{p}<$ $0.05, * \star=p<0.01$.

A

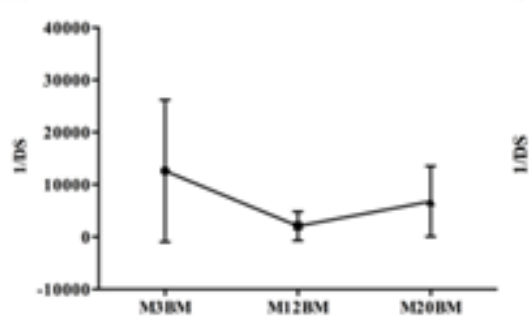

B

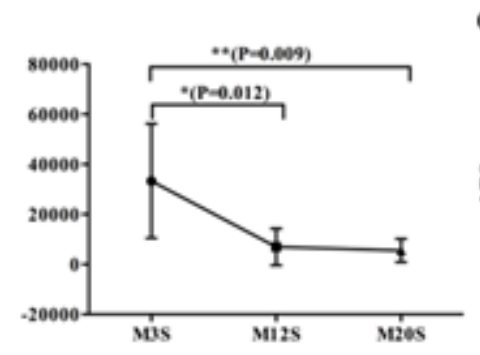

C

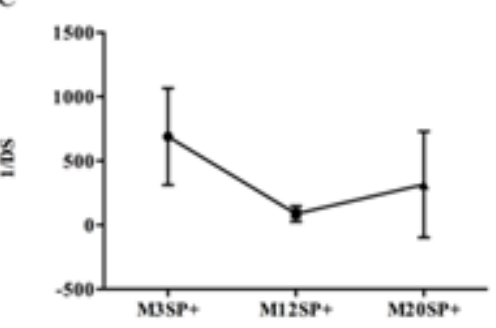

Figure 4

Diversity of the productive sequences of the BCR H-CDR3 repertoire in mice of different ages. A. Bone marrow B cell repertoire diversity. B. Spleen B cell repertoire diversity. C. Spleen memory B cell repertoire diversity. 1/DS are shown (mean $\pm S D$; error bars represent $S D$ ). The $p$ values were determined using oneway ANOVA with a Bonferroni correction. All the statistically significant differences are indicated. $*=p<$ $0.05, * \star=p<0.01$.
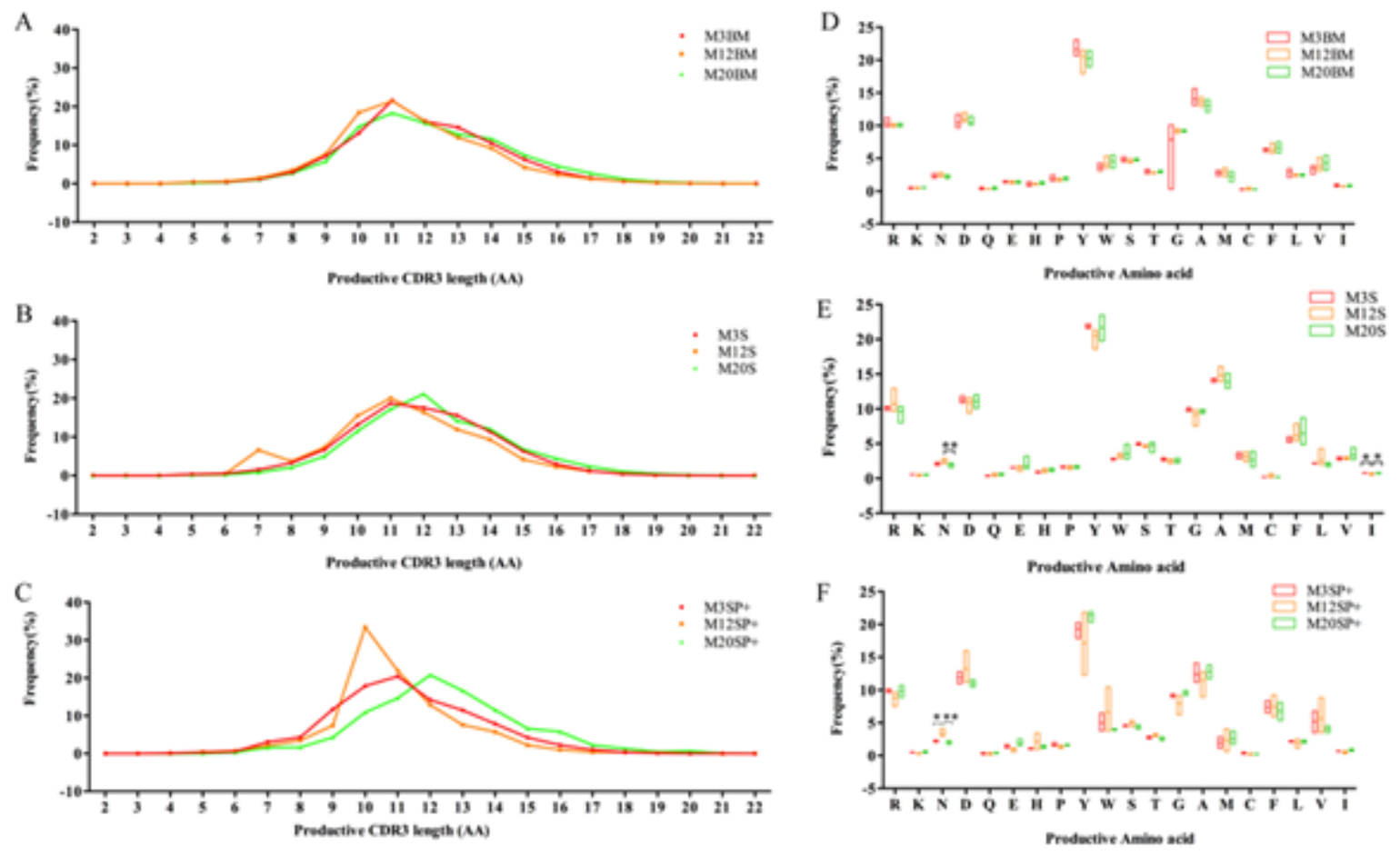

Figure 5 
Productive sequence CDR3 length and AA usage for mice of different ages.A. Bone marrow $B$ cell CDR3 length. B. Spleen B cell CDR3 length. C. Spleen memory B cell CDR3 length. D. Bone marrow B cell AA usage. E. Spleen B cell AA usage. F. Spleen memory B cell AA usage. All the statistically significant differences are indicated. ${ }^{*}=p<0.05, * *=p<0.01$.
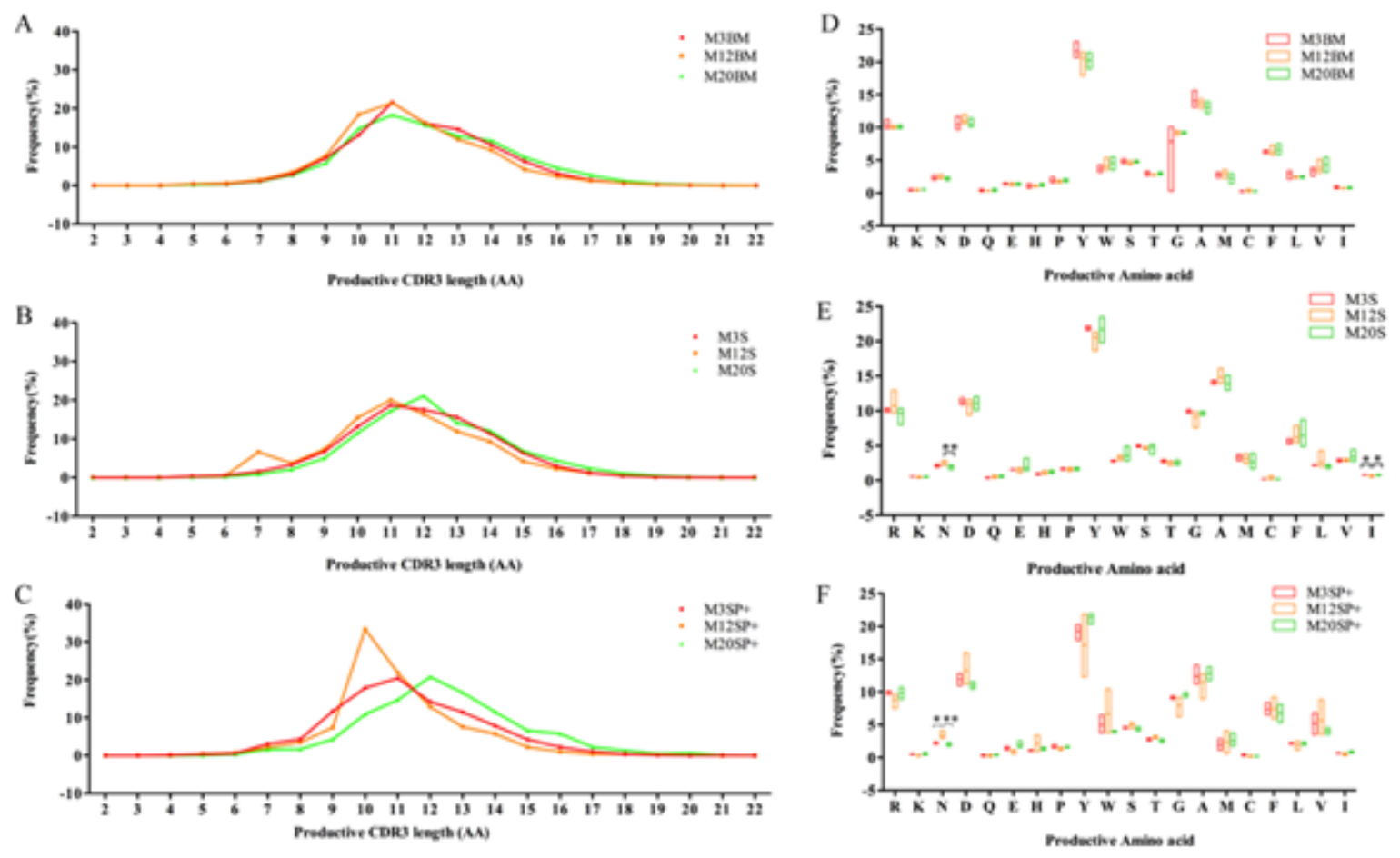

\section{Figure 5}

Productive sequence CDR3 length and AA usage for mice of different ages.A. Bone marrow $B$ cell CDR3 length. B. Spleen B cell CDR3 length. C. Spleen memory B cell CDR3 length. D. Bone marrow B cell AA usage. E. Spleen B cell AA usage. F. Spleen memory B cell AA usage. All the statistically significant differences are indicated. $*=p<0.05, * \star=p<0.01$.
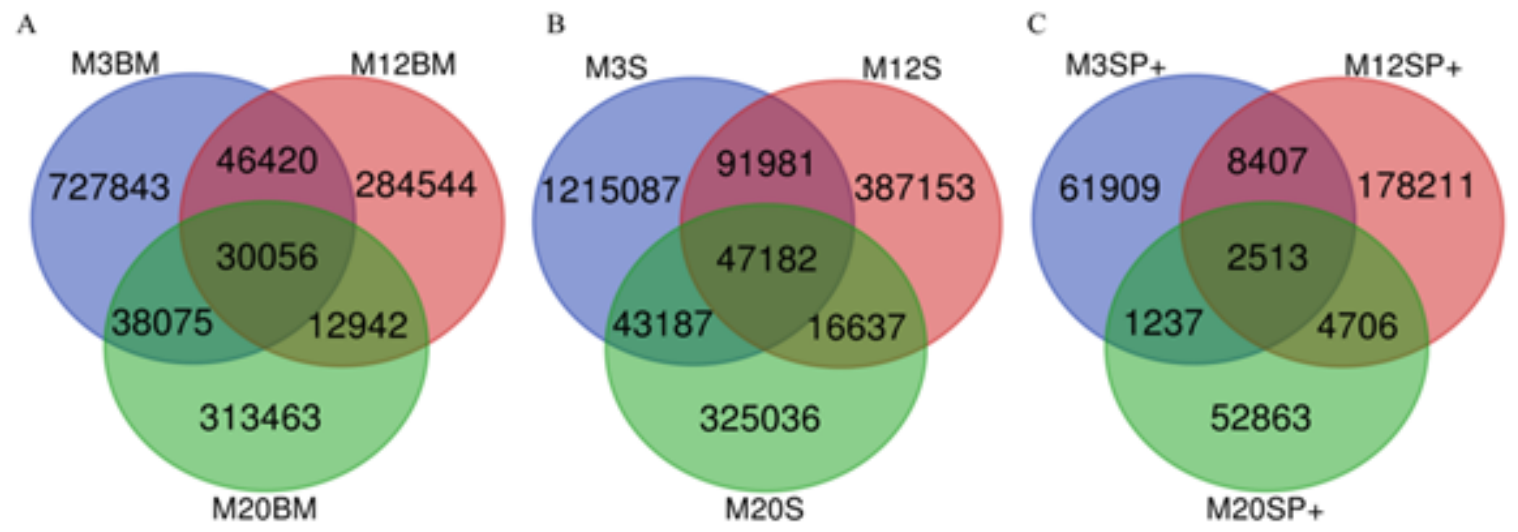

Figure 6 
Overlap amino acid sequences of the BCR H-CDR3 repertoire in mice of different ages. A. Overlap AA sequence of the CDR3 repertoire of bone marrow $B$ cells. $B$. Overlap AA sequence of the CDR3 repertoire of spleen B cells. C. Overlap AA sequence of the CDR3 repertoire of spleen memory B cells.
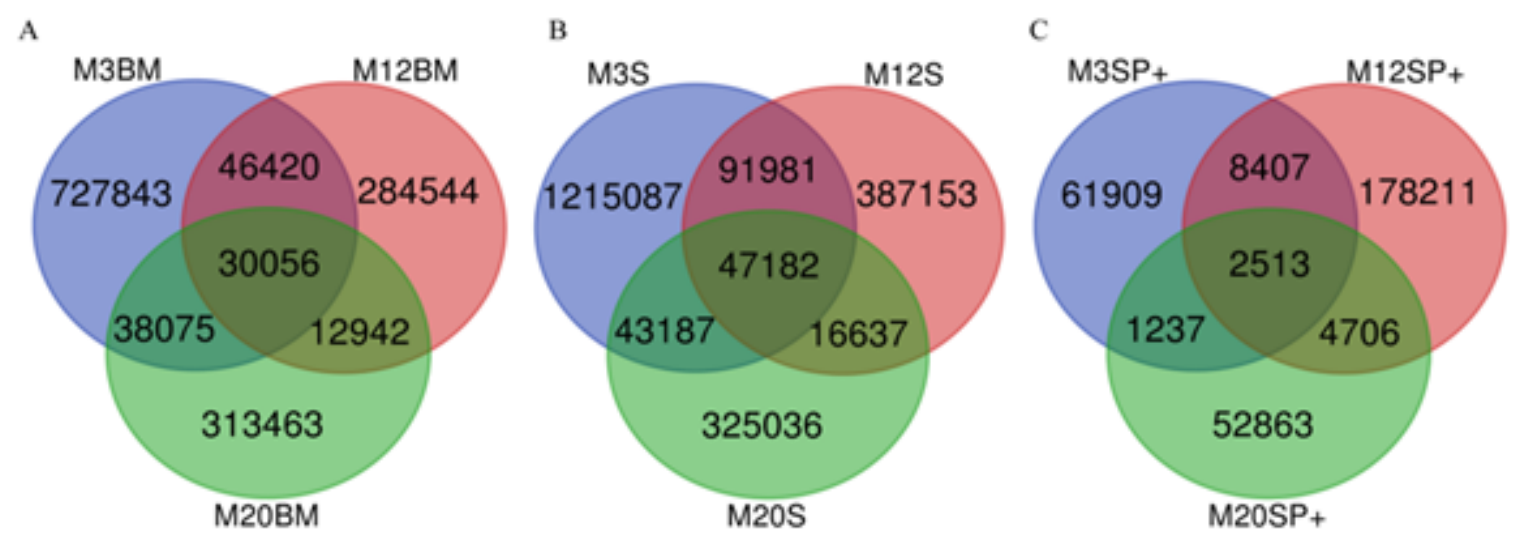

Figure 6

Overlap amino acid sequences of the BCR H-CDR3 repertoire in mice of different ages. A. Overlap AA sequence of the $C D R 3$ repertoire of bone marrow $B$ cells. B. Overlap AA sequence of the CDR3 repertoire of spleen B cells. C. Overlap AA sequence of the CDR3 repertoire of spleen memory B cells.

\section{Supplementary Files}

This is a list of supplementary files associated with this preprint. Click to download.

- Supplementaryinformation.docx

- Supplementaryinformation.docx 